\begin{tabular}{|c|l|}
\hline Title & $\begin{array}{l}\text { Electrochemical and morphological characterization of porous al umina formed by galvanostatic anodizing in etidronic } \\
\text { acid }\end{array}$ \\
\hline Author(s) & Iwai, Mana; Kikuchi, Tatsuya; Suzuki, Ryosuke O.; Natsui, Shungo \\
\hline Citation & $\begin{array}{l}\text { Electrochimica acta, 320, UNSP 134606 } \\
\text { https://doi.org/10.1016/.electacta.2019.134606 }\end{array}$ \\
\hline Issue Date & 2019-10-10 \\
\hline Doc URL & http://hdl.handle.net/2115/82854 \\
\hline Rights & $\begin{array}{l}\text { O2019. This manuscript version is made available under the CC-BY-NC-ND 4.0 license } \\
\text { http://creativecommons.org/icenses/by-nc-nd/4.0/ }\end{array}$ \\
\hline Rights(URL) & http://creativecommons.org/icenses/by-nc-nd/4.0/ \\
\hline Type & article (author version) \\
\hline Mile Information & Manuscript (Electrochimica).pdf \\
\hline
\end{tabular}

Instructions for use 


\section{Electrochemical and Morphological Characterization of Porous Alumina Formed by Galvanostatic Anodizing in Etidronic Acid}

Mana Iwai ${ }^{1}$, Tatsuya Kikuchi ${ }^{1, *}$, Ryosuke O. Suzuki ${ }^{1}$, Shungo Natsui ${ }^{2}$

${ }^{1}$ Faculty of Engineering, Hokkaido University, N13-W8, Kita-ku, Sapporo, Hokkaido, 060-8628, Japan

${ }^{2}$ Institute of Multidisciplinary Research for Advanced Materials, Tohoku University, Katahira 2-1-1, Aoba-ku, Sendai, 980-8577, Japan

*Corresponding author: Tatsuya Kikuchi

e-mail: kiku@eng.hokudai.ac.jp 


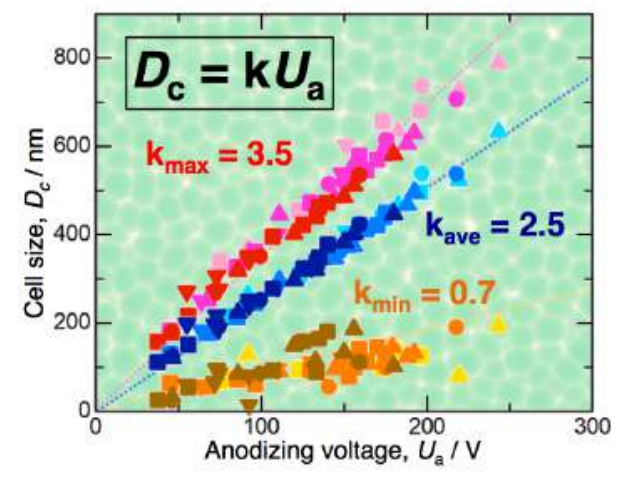

Graphical Abstract 


\begin{abstract}
Electrochemical and morphological characterization of the porous alumina formed by galvanostatic anodizing in etidronic acid under various operating conditions was performed. High-purity aluminum plates were anodized in 0.03-3 $\mathrm{M}$ etidronic acid solutions at 273-333 $\mathrm{K}$ and $0.25-500 \mathrm{Am}^{-2}$ for up to $24 \mathrm{~h}$. Galvanostatic anodizing in etidronic acid operated over a wide range voltage measuring from a few $\mathrm{V}$ to $246 \mathrm{~V}$. The time required for the steady growth of porous alumina not only depends on the current density but also the temperature and the concentration of the electrolyte solution during galvanostatic anodizing. The average, maximum, and minimum cell sizes of the porous alumina were directly proportional to the anodizing voltage with a proportionality constant of 2.5, 3.5 and 0.7, respectively, and were independent of other parameters. The number density of the cell was also a function of the anodizing voltage and agreed with the theoretical value obtained for ordered porous alumina with an ideal honeycomb distribution. The maximum voltage measured during galvanostatic anodizing was linearly proportional to the plateau voltage with a proportionality constant of 1.4.
\end{abstract}

Keywords: Anodizing; Aluminum; Etidronic Acid; Porous Alumina; Morphology 


\section{Introduction}

Anodic porous alumina with numerous nanoscale pores can be fabricated by anodizing aluminum in appropriate acidic or alkaline solutions [1-4] and has been widely used for various applications from traditional corrosion protection [5-7] to current nanoscience and engineering applications [8-10]. The nanomorphology of porous alumina, such as the pore size, interval, regularity, and aspect ratio, strongly depends on the electrolyte species and electrochemical conditions during anodizing [11-13]. Therefore, the fabrication of advanced porous alumina with a novel pore structure and arrangement has been investigated with new electrolytes [14-17], hard anodizing [18-20], and a pulsed technique [21-23] to expand the applicability of porous alumina.

Etidronic acid (1-hydroxyethane-1,1-diphosphonic acid, $\mathrm{C}_{2} \mathrm{H}_{8} \mathrm{O}_{7} \mathrm{P}_{2}$ ) is currently one of the most popular chelating agent [24] and has been widely used in various applications, such as food additives, cosmetics, detergents, corrosion inhibitors, osteoporosis medicines, and water treatment agents $[25,26]$. Very recently, etidronic acid was reported to possess the potential to behave as a suitable acidic electrolyte for the fabrication of porous alumina by anodizing aluminum [27]. Ordered porous alumina with a large-scale cell (interpore distance) measuring 400-670 $\mathrm{nm}$ can be easily fabricated via two-step potentiostatic anodizing in etidronic acid at high voltages of more than 200 V. [27-29]. Hard anodic coatings with a high Vickers hardness measuring $\mathrm{Hv}=610-769$ can be achieved by anodizing in low concentration etidronic acid at low temperature due to their low porosity [30]. Advanced hard anodic coatings with microarc oxide/porous alumina double layers were successfully developed by inducing a steady plasma spark during etidronic acid anodizing at high voltages [31]. The fabrication of novel ordered nanomaterials, such as submicrometer-scale convex lens arrays [32], structural color devices [33], antireflective surfaces [34,35], and titanium dioxide nanocolumns [36], has been demonstrated through etidronic acid anodizing.

Porous alumina can be fabricated by anodizing aluminum at either a constant cell voltage (potentiostatic) or constant current density (galvanostatic) [1]. Potentiostatic anodizing in etidronic acid at high voltages has been reported for the formation of large-scale ordered porous alumina. Although galvanostatic anodizing is typically used in industrial applications for surface finishing, very little has been reported on electrochemical behavior during galvanostatic anodizing in etidronic acid and the nanostructure.

In the present investigation, we examine galvanostatic anodizing in etidronic acid under various operating parameters, such as the concentration, temperature, and current density. Stable anodizing conditions and their corresponding voltages were examined by electrochemical measurements. In addition, nanostructural characterization of the porous alumina was carried out by electron microscopy and subsequent image analysis.

\section{Experimental}




\subsection{Pretreatment of aluminum specimens}

High-purity aluminum plates (purity: $99.999 \mathrm{wt} \%$, thickness: $500 \mu \mathrm{m}$, Nippon Light Metal, Japan) were used as the anodizing specimens. The aluminum plates were cut into square pieces $(20 \mathrm{~mm} \times 20 \mathrm{~mm})$ with a handle $(12 \mathrm{~mm} \times 8 \mathrm{~mm})$ for electrical contact. The aluminum specimens were ultrasonically cleaned in ethanol for $10 \mathrm{~min}$ at room temperature. The lower half of the handle of the specimen was then covered with a silicone resin (KE45W, Shin-Etsu, Japan) to prevent contact with the electrolyte solution. After resin coating, the specimens were electrochemically polished in a 78 vol $\% \mathrm{CH}_{3} \mathrm{COOH} / 22 \mathrm{vol} \% 70 \%-\mathrm{HClO}_{4}$ solution $(280 \mathrm{~K})$ at a constant voltage of $28 \mathrm{~V}$ for $1 \mathrm{~min}$.

\subsection{Galvanostatic anodizing in etidronic acid}

An electrochemical cell with an inner diameter of $55 \mathrm{~mm}$ was used for anodizing. An electropolished aluminum specimen as the anode and a platinum plate (purity: 99.95 wt $\%$, thickness: $100 \mu \mathrm{m}$, Furuya Metal, Japan) as the cathode were immersed in 0.03-3 $\mathrm{M}$ etidronic acid solutions (volume: $150 \mathrm{~mL}$, Tokyo Chemical Industry, Japan) at 273-333 K. The aluminum anode and platinum cathode were $22 \mathrm{~mm}$ apart and parallel, and the solution was stirred with a magnetic stirrer. The aluminum specimens were galvanostatically anodized at $0.25-500 \mathrm{Am}^{-2}$ for up to $24 \mathrm{~h}$ using a direct power supply (PWR400H, Kikusui Electronics, Japan, and MODEL 6911, Metronix, Japan). The anodizing voltage was measured with a digital multimeter (VOAC7602, IWATSU, Japan, and DMM4040, Tektronix, USA) during galvanostatic anodizing. The temperature of the solution was maintained with a constant temperature water bath (UCT-1000A, AS ONE, Japan).

After anodizing, the specimens were immersed in a $0.20 \mathrm{M} \mathrm{CrO}_{3} / 0.51 \mathrm{M} \mathrm{H}_{3} \mathrm{PO}_{4}$ solution $(353 \mathrm{~K})$ to dissolve the porous alumina film, and the aluminum dimple array corresponding to the growth interface of the porous alumina was exposed to the surface.

\subsection{Morphological characterization}

The nanostructures of the porous alumina and the aluminum dimple array were observed by field-emission scanning electron microscopy (FE-SEM, JSM6500F, JEOL, Japan). Before the SEM observations, a thin platinum electroconductive layer was coated on the specimens by a sputter coater (MSP-1S, Vacuum Device, Japan). The cell size of the porous alumina was quantified using image analysis software (Image-Pro 10, Media Cybernetics, USA).

\section{Results and Discussion}

3.1 Steady state plateau voltage during galvanostatic anodizing in etidronic acid

The electrochemical behaviors during galvanostatic anodizing in etidronic acid were investigated in detail. Figure 1a shows the voltage-time curves during galvanostatic anodizing in a $c=0.3 \mathrm{M}$ etidronic acid solution at $T_{\mathrm{a}}=298 \mathrm{~K}$. At current 
densities of $i_{\mathrm{a}}=1.25-20 \mathrm{Am}^{-2}$, typical voltage-time curves for the formation of uniform porous alumina were obtained. The voltage linearly increased with the anodizing time in the initial stage, reached a maximum value, gradually decreased, and then reached a plateau value. Figure $1 \mathrm{~b}$ shows the surface appearance and corresponding SEM image of the specimen anodized at $10 \mathrm{Am}^{-2}$ for $60 \mathrm{~min}$, and a uniform, light-gray porous alumina film with disordered nanoscale pores measuring approximately $100 \mathrm{~nm}$ in diameter was obtained over the entire aluminum surface. The plateau voltage corresponding to the steady growth of the porous alumina increased with the current density, and a notably high plateau voltage measuring $195 \mathrm{~V}$ was achieved at $20 \mathrm{Am}^{-2}$. However, the excess current density at $50 \mathrm{Am}^{-2}$ caused oxide burning with an unstable oscillation in the voltage-time curve, and a nonuniform dark brown oxide film with many cracks was formed on the surface (Fig. 1c). Therefore, stable formation of the porous alumina film can be achieved below $20 \mathrm{Am}^{-2}$ in a $0.3 \mathrm{M}$ etidronic acid solution at $293 \mathrm{~K}$. At this concentration and temperature, the plateau voltage can be controlled over a wide range up to $195 \mathrm{~V}$ by choosing the appropriate current density.

The effect of various anodizing parameters, such as the solution concentration, temperature, and current density, on the steady state plateau voltage during galvanostatic anodizing in etidronic acid was investigated. Figure 2a summarizes the relationship between the plateau voltage, $U_{\mathrm{p}}$, and the current density, $i_{\mathrm{a}}$, during anodizing in a $0.03 \mathrm{M}$ etidronic acid solution. Here, the plateau voltage was defined as the point at which the change in the anodizing voltage was within $0.3 \mathrm{~V}$ per minute at the stage of the porous alumina formation. At a lower solution temperature of $293 \mathrm{~K}$, the plateau voltage increased with the current density, and the highest plateau voltage measuring $246 \mathrm{~V}$ was achieved. However, the current density at this highest voltage was extremely low at $15 \mathrm{Am}^{-2}$, and applying a higher current density of more than $20 \mathrm{Am}^{-2}$ caused oxide burning with unstable voltage oscillation. As the temperature increased to 313 and $333 \mathrm{~K}$, the plateau voltage significantly decreased to approximately more than $100 \mathrm{~V}$ at the same current density due to thinning of the barrier layer during anodizing at high temperature. In addition, the oxide burning region moved to lower voltages as the temperature increased. On the other hand, a higher current density at $100 \mathrm{Am}^{-2}$ could be applied at $333 \mathrm{~K}$ without oxide burning. Similar tendencies were observed as the concentration increased to $0.3 \mathrm{M}$ (Fig. 2b) and $3 \mathrm{M}$ (Fig. 2c). In each concentration, the shape of the plateau voltage vs. current density was nearly linear with a slight s-shape. The plateau voltage at the same temperature and current density decreased with the concentration of the etidronic acid solution due to the higher solubility of the barrier layer at higher concentrations.

During galvanostatic anodizing, porous alumina grows through barrier oxide formation in the initial transition period. The time until the plateau voltage is achieved, i.e., the time required for the steady growth of porous alumina, was measured during anodizing under various operating conditions. Figure 3 shows the change in the time required to reach steady growth, $t_{\mathrm{pla}}$, with the current density, $i_{\mathrm{a}}$, during galvanostatic 
anodizing in a) $0.03 \mathrm{M}$, b) $0.3 \mathrm{M}$, and c) $3 \mathrm{M}$ etidronic acid solutions. The required time decreased with the increasing current density at each concentration due to the high growth rate of the anodic oxide during anodizing at high current density. However, at the same current density, the required time increased as the solution temperature decreased. In addition, the required time increased as the concentration decreased at the same current density. Therefore, the time required for the steady growth of porous alumina depends not only on the current density but also on the temperature and the concentration of the electrolyte solution during galvanostatic anodizing.

Based on the electrochemical measurements, galvanostatic anodizing in etidronic acid operates over an extremely wide range voltage from a few V to $246 \mathrm{~V}$. This voltage range is much higher than that obtained for galvanostatic anodizing without oxide burning in typical electrolytes, such as sulfuric acid, measuring up to approximately 20 $\mathrm{V}$ [37]; and oxalic acid, measuring up to approximately $45 \mathrm{~V}$ [38]. Because the cell size of porous alumina increases with the anodizing voltage, various porous alumina films may easily be fabricated by anodizing in etidronic acid. However, galvanostatic anodizing causes the growth of disordered porous alumina with different cell sizes. Therefore, the cell distribution of the porous alumina formed by galvanostatic anodizing is discussed in detail in the next section.

\subsection{Cell distribution of porous alumina}

When the porous alumina film was dissolved from the aluminum surface in a $\mathrm{CrO}_{3} / \mathrm{H}_{3} \mathrm{PO}_{4}$ solution, the growth interface between the porous alumina and the aluminum substrate was exposed to the surface as an aluminum dimple array. Figure $4 \mathrm{a}$ shows SEM images of the exposed aluminum surface after galvanostatic anodizing in a $0.3 \mathrm{M}$ etidronic acid solution at $293 \mathrm{~K}$ and $10-20 \mathrm{Am}^{-2}$ for $60 \mathrm{~min}$. Disordered dimple arrays with different polygonal dimples, including tetragonal, pentagonal, hexagonal, and heptagonal, were distributed on the entire surface. The diameter of the dimples, i.e., the cell size of the porous alumina, seemed to increase with the current density due to the increasing anodizing voltage $\left(i_{\mathrm{a}}=10 \mathrm{Am}^{-2}\right.$ at $U_{\mathrm{a}}=156 \mathrm{~V}, 15 \mathrm{Am}^{-2}$ at $182 \mathrm{~V}$, and 20 $\mathrm{Am}^{-2}$ at $\left.195 \mathrm{~V}\right)$.

Unlike ordered porous alumina, the cell size, $D_{\mathrm{c}}$, of the porous alumina formed by galvanostatic anodizing is not easily calculated due to the irregularity of the cells. Here, the cell size was calculated from the SEM images of the disordered dimple array using image analysis software as follows: a) A projected area of each cell was measured from the SEM image. b) We considered a regular hexagon possessing the same calculated area. c) A short diagonal of the regular hexagon was geometrically calculated, and the result equals the cell size. The normalized cell size histograms of the porous alumina are shown in Figure $4 \mathrm{~b}$. At $i_{\mathrm{a}}=10 \mathrm{Am}^{-2}$, the histogram possessed a considerably left-skewed distribution without a symmetric shape, and the average cell size was calculated to be $374 \mathrm{~nm}$. Similar left-skewed distributions were obtained by anodizing at higher current densities of $15 \mathrm{Am}^{-2}$ and $20 \mathrm{Am}^{-2}$, and the average cell size increased 
with the current density ( $449 \mathrm{~nm}$ and $497 \mathrm{~nm}$, respectively).

Figure 5a-5c shows the relationship between the anodizing voltage, $U_{\mathrm{a}}$, and the

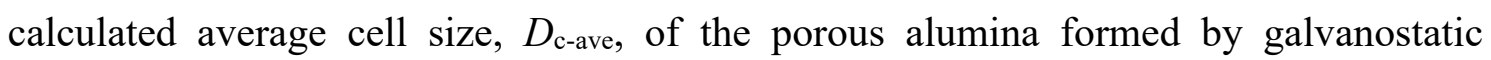
anodizing in a) $0.03 \mathrm{M}$, b) $0.3 \mathrm{M}$, and c) $3 \mathrm{M}$ etidronic acid solutions at various temperatures and current densities. It is clear that the average cell size linearly increased with the anodizing voltage at all concentrations. Figure $5 \mathrm{~d}$ summarizes the average cell size vs. anodizing voltage for all concentrations, and the dashed line shows the linear relation obtained by the least-squares method. There were similar linear relationships with no dependence on the concentration and temperature. Namely, the average cell size depends only on the anodizing voltage and is directly proportional to the anodizing voltage with a proportionality constant of 2.5 .

$$
D_{\text {c-ave }}=2.5 U_{\mathrm{a}}[\mathrm{nm}]
$$

Several research groups have previously reported that self-ordered porous alumina formed by potentiostatic anodizing exhibits a cell size voltage dependence with a proportionality constant of 2.5 , and the cell size can be accurately controlled by adjusting the voltage during anodizing [1,2,39]. Although galvanostatic anodizing causes the formation of disordered porous alumina with a wide ranging cell distribution, as shown in Figure 4, the average cell size formed by galvanostatic anodizing also has the same proportional relation with a proportionality constant of 2.5.

The maximum cell size, $D_{\text {c-max }}$, and the minimum cell size, $D_{\text {c-min, of the }}$ disordered porous alumina were also measured under each operating condition, and the relationship of the cell size vs. the anodizing voltage is summarized in Figure 6. Similar to the average cell size, the maximum and minimum cell sizes were directly proportional to the anodizing voltage with proportionality constants of 3.5 and 0.7 , respectively.

$$
\begin{aligned}
D_{\text {c-max }} & =3.5 U_{\mathrm{a}}[\mathrm{nm}] \\
D_{\text {c-min }} & =0.7 U_{\mathrm{a}}[\mathrm{nm}]
\end{aligned}
$$

Therefore, the disordered porous alumina film formed by galvanostatic anodizing contains cells up to 1.4 times larger and 0.28 times smaller than the average cells in the porous layer matrix. Importantly, these average, maximum, and minimum cell sizes are determined by the anodizing voltage and are independent of other operating factors, such as the solution temperature, concentration, and current density, during anodizing.

The number of cells formed in the porous alumina was calculated, and Figure 7 summarizes the changes in the number density of the cells, $N_{\mathrm{c}}$, with the anodizing voltage, $U_{\mathrm{a}}$. Here, a dashed line indicates the theoretical number density of the cell formed in the ideal honeycomb porous alumina, $N_{\text {c-ideal, }}$ which is geometrically defined as follows:

$$
N_{\text {c-ideal }}=\left(2 \times 10^{18}\right) /\left(\sqrt{ } 3 \times D^{2} \text { c-ideal }\right)\left[\mathrm{m}^{-2}\right]
$$

where $D_{\text {c-ideal }}$ corresponds to the cell size of the ordered porous alumina. The $D_{\text {c-ideal }}$ value is linearly proportional to the anodizing voltage with the proportionality constant 2.5: 


$$
D_{\text {c-ideal }}=2.5 U_{\mathrm{a}}[\mathrm{nm}]
$$

Therefore, Equation (4) can be rewritten:

$$
N_{\text {c-ideal }}=\left(3.2 \times 10^{17}\right) /\left(\sqrt{ } 3 \times U^{2}\right)\left[\mathrm{m}^{-2}\right]
$$

The experimental number densities obtained by galvanostatic anodizing at various concentrations and various temperatures were measured to be on the order of $10^{12}-10^{14}$ $\mathrm{m}^{-2}$ in the range of $37-246 \mathrm{~V}$, and these values were in good agreement with the ideal dashed curve. Namely, the number density of the porous alumina cell formed by galvanostatic anodizing depends only on the anodizing voltage and is independent of other operating factors.

In summary, the nanomorphologies of porous alumina, such as the average, maximum, and minimum cell sizes, and the number density are determined by the anodizing voltage and can be accurately controlled by adjusting the voltage during galvanostatic anodizing.

\subsection{Relationship between the maximum and plateau voltages}

During galvanostatic anodizing to form porous alumina, the voltage linearly increases, exhibits a maximum value, and then reaches a plateau after a gradual decrease (Fig. 1). Here, we found that there is a unique proportional relation between the maximum and plateau voltages. Figure 8 shows the changes in the maximum voltage, $U_{\max }$, with the plateau voltage, $U_{\mathrm{p}}$, during galvanostatic anodizing under various operating conditions. It was clear that the maximum voltage was linearly proportional to the plateau voltage with a proportionality constant of 1.4 , as follows:

$$
U_{\max }=1.4 U_{\mathrm{p}}[\mathrm{V}]
$$

Similar to the porous alumina cell morphology shown in Figures 5, 6, and 7, the maximum voltage only depends on the plateau voltage and is independent of the other operating factors.

Wood et al. have reported the anodizing behaviors of aluminum in sulfuric acid solutions under a variety of conditions with various current densities and concentrations [40]. Although they did not discuss the relationship between the maximum voltage and the plateau voltage, there was no linear relation between these values (the maximum voltage/plateau voltage ratio: approximately 1.00-1.43). In later years, Thompson et al. have reported the galvanostatic anodizing of aluminum alloys such as AA2024 and AA7075 in sulfuric acid [41], and the maximum peak disappeared on the voltage-time curve obtained by anodizing of aluminum alloys. On the other hand, Gastón-García et al. have reported that clear maximum peaks were obtained by galvanostatic anodizing of AA1050 aluminum alloy in sulfuric acid [42]. Therefore, it seems that the shape of the voltage-time curve measured by typical anodizing strongly depends on the aluminum substrate and the electrolyte species used.

The voltage evolution from the maximum value to the plateau usually corresponds to the morphological transition from barrier oxide growth to porous alumina growth [1]. Therefore, a proportional relationship between the maximum and 
plateau voltages may be strongly related to the morphological change of the anodic oxide. Chu et al. have reported that the thickness of the barrier layer, $\delta$, at the bottom of porous alumina formed in various electrolyte solutions is determined as follows [43]:

$$
\delta_{\mathrm{b}}=1.0 U_{\mathrm{a}}[\mathrm{nm}]
$$

Because the porous layer was already formed in the anodic oxide before the voltage reached the maximum value, the voltage decrease from the maximum value to the plateau may correspond to a decrease in the barrier layer thickness. However, the reason why the maximum voltage decreased to the plateau voltage during the initial stage of anodizing is unclear, and further investigation is required.

\section{Conclusions}

We have demonstrated galvanostatic anodizing in etidronic acid under a variety of conditions with various current densities, concentrations, and temperatures. In addition, the morphology of the porous alumina cell was examined by SEM and image analyses. The following conclusions can be drawn from our investigation.

1) Galvanostatic anodizing in etidronic acid can operate over a wide voltage range measuring from a few $\mathrm{V}$ to $246 \mathrm{~V}$.

2) The time required for steady growth decreases with increasing current density. In addition, the time strongly depends on the temperature and the concentration of the solution.

3) The histogram of the cell size formed in the disordered porous alumina has a left-skewed distribution without a symmetric shape. The average, maximum, and minimum cell sizes are directly proportional to the anodizing voltage with a proportionality constant of $2.5,3.5$ and 0.7 , respectively.

4) The number density of the disordered porous alumina cell is only a function of the anodizing voltage, which is in good agreement with the resultant ordered porous alumina with an ideal honeycomb distribution.

5) The maximum voltage is linearly proportional to the plateau voltage with a proportionality constant of 1.4 .

\section{Acknowledgments}

This research was funded by the Light Metal Education Foundation, Japan, and the Japan Society for the Promotion of Science (JSPS) "KAKENHI". The authors thank Mr. Nobuyuki Miyazaki and Dr. Takashi Endo (Hokkaido University) for their help with electron microscopy through the Nanotechnology Platform Program of the Ministry of Education, Culture, Sports, Science, and Technology (MEXT), Japan. The authors also thank Dr. Ken Ebihara (Nippon Light Metal) for his valuable advice in the discussion.

\section{References}

1) W. Lee, D. J. Park, Porous anodic aluminum oxide: anodization and templated synthesis of functional nanostructures, Chem. Rev. 114 (2014) 7487-7556. 
2) G.D. Sulka, Highly ordered anodic porous alumina formation by self-organized anodizing, in: A. Eftekhari (Ed.), Nanostructured Materials in Electrochemistry, Wiley-VCH, 2008, p. 1.

3) J.E. Houser, K.R. Hebert, The role of viscous flow of oxide in the growth of self-ordered porous anodic alumina films, Nat. Mater. 8 (2009) 415-420.

4) K.R. Hebert, S.P. Albu, I. Paramasivam, P. Schmuki, Morphological instability leading to formation of porous anodic oxide films, Nat. Mater. 8 (2012) 162-166.

5) T. He, Y. Wang, Y. Zhang, T. Xu, T. Liu, Super-hydrophobic surface treatment as corrosion protection for aluminum in seawater, Corr. Sci. 51 (2009) 1757-1761.

6) X. Li, X. Nie, L. Wang, D. O. Northwood, Corrosion protection properties of anodic oxide coatings on an Al-Si alloy, Surf. Coat. Technol. 200 (2005) 1994-2000.

7) L. H. Chiu, C. C. Chen, C. F. Yang, Improvement of corrosion properties in an aluminum-sprayed AZ31 magnesium alloy by a post-hot pressing and anodizing treatment. Surf. Coat. Technol. 191 (2005) 181-187.

8) T. Yanagishita, K. Nishio, H. Masuda, Fabrication of Metal Nanohole Arrays with High Aspect Ratios Using Two-Step Replication of Anodic Porous Alumina, Adv. Mater. 17 (2005) 2241-2243.

9) T. Yanagishita, H. Masuda, High-throughput fabrication process for highly ordered through-hole porous alumina membranes using two-layer anodization, Electrochim. Acta 184 (2015) 80-85.

10) H. Q. Cao, Y. Xu, J. M. Hong, H. B. Liu, G. Yin, B. L. Li, C. Y. Tie, Z. Xu, Sol-gel template synthesis of an array of single crystal $\mathrm{CdS}$ nanowires on a porous alumina template, Adv. Mater. 13 (2001) 1393-1394.

11) H. Masuda, K. Yada, A. Osaka, Self-ordering of cell configuration of anodic porous alumina with large-size pores in phosphoric acid solution, Jpn. J. Appl. Phys. 37 (1998) L1340.

12) F. Li, L. Zhang, R. M. Metzger, On the growth of highly ordered pores in anodized aluminum oxide, Chem. Mater. 10 (1998) 2470-2480.

13) R. L. Chiu, P. H. Chang, C. H. Tung, The effect of anodizing temperature on anodic oxide formed on pure Al thin films, Thin Solid Films 260 (1995) 47-53.

14) T. Kikuchi, T. Yamamoto, S. Natsui, R. O. Suzuki, Fabrication of anodic porous alumina by squaric acid anodizing, Electrochim. Acta 123 (2014) 14-22.

15) T. Kikuchi, O. Nishinaga, S. Natsui, R. O. Suzuki, Self-ordering behavior of anodic porous alumina via selenic acid anodizing, Electrochim. Acta 137 (2014) 728-735.

16) T. Kikuchi, D. Nakajima, J. Kawashima, S. Natsui, R. O. Suzuki, Fabrication of anodic porous alumina via anodizing in cyclic oxocarbon acids, Appl. Surf. Sci. 313 (2014) 276-285.

17) S. Akiya, T. Kikuchi, S. Natsui, N. Sakaguchi, R.O. Suzuki, Self-ordered porous alumina fabricated via phosphonic acid anodizing, Electrochim. Acta 190 (2016) 471-479.

18) W. Lee, J. C. Kim, U. Gösele, Spontaneous current oscillations during hard 
anodization of aluminum under potentiostatic conditions, Adv. Funct. Mater. 20 (2010) 21-27.

19) K. Schwirn, W. Lee, R. Hillebrand, M. Steinhart, K. Nielsch, U. Gösele, Self-ordered anodic aluminum oxide formed by $\mathrm{H}_{2} \mathrm{SO}_{4}$ hard anodization, ACS Nano 2 (2008) 302-310.

20) W. Lee, R. Ji, U. Gösele, K. Nielsch, Fast fabrication of long-range ordered porous alumina membranes by hard anodization, Nat. Mater. 5 (2006) 741-747.

21) W. Lee, J. C. Kim, Highly ordered porous alumina with tailor-made pore structures fabricated by pulse anodization, Nanotechnol. 21 (2010), 485304.

22) W. Lee, K. Schwirn, M. Steinhart, E. Pippel, R. Scholz, U. Gösele, Structural engineering of nanoporous anodic aluminium oxide by pulse anodization of aluminium, Nat. Nanotechnol. 3 (2008) 234.

23) J. Martín, M. Martín-González, J.F. Fernández, O. Caballero-Calero, Ordered three-dimensional interconnected nanoarchitectures in anodic porous alumina, Nat. Commun. 5 (2014) 5130.

24) G. De-Deus, M. Zehnder, C. Reis, S. Fidel, R. A. S. Fidel, J. Galan Jr, S. Paciornik, Longitudinal co-site optical microscopy study on the chelating ability of etidronate and EDTA using a comparative single-tooth model, J. Endod. 34 (2008) 71-75.

25) M. T. Arias-Moliz, R. Ordinola-Zapata, P. Baca, M. Ruiz-Linares, C. M. Ferrer-Luque, Antimicrobial activity of a sodium hypochlorite/etidronic acid irrigant solution, J. Endod. 40 (2014) 1999-2002.

26) K. Airey, R. D. Armstrong, T. Handyside, The use of molybdates combined with etidronic acid as corrosion inhibitors for iron, Corr. Sci. 28 (1988) 449-460.

27) T. Kikuchi, O. Nishinaga, S. Natsui, R.O. Suzuki, Fabrication of self-ordered porous alumina via etidronic acid anodizing and structural color generation from submicrometer-scale dimple array, Electrochim. Acta 156 (2015) 235-243.

28) A. Takenaga, T. Kikuchi, S. Natsui, R.O. Suzuki, Exploration for the self-ordering of porous alumina fabricated via anodizing in etidronic acid, Electrochim. Acta 211 (2016) 515-523.

29) M. Sepúlveda, J.G. Castaño, F. Echeverría, Influence of temperature and time on the fabrication of self-ordering porous alumina by anodizing in etidronic acid, Appl. Surf. Sci. 454 (2018) 210-217.

30) T. Kikuchi, A. Takenaga, S. Natsui, R.O. Suzuki, Advanced hard anodic alumina coatings via etidronic acid anodizing, Surf. Coat. Technol. 326 (2017) 72-78.

31) H. Huang, J. Qiu, M. Sun, W. Liu, X. Wei, E. Sakai, K. Ito, A hard coating with $\mathrm{MAO} / \mathrm{AAO}$ double layers prepared on aluminum in etidronic acid by DC oxidation, Surf. Coat. Technol. 360 (2019) 307-317.

32) K. Kawahara, T. Kikuchi, S. Natsui, R.O. Suzuki, Fabrication of ordered submicrometer-scale convex lens array via nanoimprint lithography using an anodized aluminum mold, Microelectron. Eng. 185 (2018) 61-68.

33) T. Kikuchi, O. Nishinaga, S. Natsui, R.O. Suzuki, Polymer nanoimprinting using an 
anodized aluminum mold for structural coloration, Appl. Surf. Sci. 341 (2015) 19-27.

34) M. Norek, M. Łażewski, Manufacturing of highly ordered porous anodic alumina with conical pore shape and tunable interpore distance in the range of $550 \mathrm{~nm}$ to 650 nm, Mater. Sci.-Poland 35 (2017) 511-518.

35) M. Norek, M. Włodarski, Morphological and chemical characterization of highly ordered conical-pore anodic alumina prepared by multistep citric acid anodizing and chemical etching process, J. Porous Mater. 25 (2018) 45-53.

36) M. Sepúlveda, J.G. Castaño, F. Echeverría, Fabrication of highly-ordered $\mathrm{TiO}_{2}$ nanocolumns by two-step anodizing of an $\mathrm{Al} / \mathrm{Ti}$ layer in etidronic acid, Mater. Chem. Phys. 216 (2018) 51-57.

37) K. Ebihara, H. Takahashi, M. Nagayama, Structure and Density of Anodic Oxide Films Formed on Aluminum in Sulfuric Acid Solutions, J. Surf. Finish. Soc. Jpn. 33 (1982) 156-164.

38) K. Ebihara, H. Takahashi, M. Nagayama, Structure and Density of Anodic Oxide Films Formed on Aluminum in Oxalic Acid Solutions, J. Surf. Finish. Soc. Jpn. 34 (1983) 548-553

39) T. Kikuchi, D. Nakajima, O. Nishinaga, S. Natsui, R.O. Suzuki, Porous aluminum oxide formed by anodizing in various electrolyte species, Curr. Nanosci. 11 (2015) 560-571.

40) G.C. Wood, J. P. O'sullivan, The anodizing of aluminum in sulphate solutions, Electrochim. Acta 15 (1970) 1865-1876.

41) M.S. de Miera, M. Curioni, P. Skeldon, G.E. Thompson, Modelling the anodizing behaviour of aluminium alloys in sulphuric acid through alloy analogues, Corr. Sci. 50 (2008) 3410-3415.

42) B. Gastón-García, García-Lecina, J.A. Díez, M. Belenguer, C. Müller, Local burning phenomena in sulfuric acid anodizing: analysis of porous anodic alumina layers on AA1050, Electrochem. Solid-State Lett. 13 (2010) C33-C35.

43) S.Z. Chu, K. Wada, S. Inoue, M. Isogai, Y. Katsuta, A. Yasumori, Large-scale fabrication of ordered nanoporous alumina films with arbitrary pore intervals by critical-potential anodization, J. Electrochem. Soc. 153 (2006) B384-B391. 


\section{Figure Captions}

Figure 1 a) Voltage-time curves during galvanostatic anodizing of aluminum in a $0.3 \mathrm{M}$ etidronic acid solution at 293 K. b), c) Surface appearance and the corresponding SEM image of the specimen anodized at b) $10 \mathrm{Am}^{-2}$ and c) $50 \mathrm{Am}^{-2}$ for $60 \mathrm{~min}$.

Figure 2 Changes in the plateau voltage, $U_{\mathrm{p}}$, with the current density, $i_{\mathrm{a}}$, during galvanostatic anodizing in a) $0.03 \mathrm{M}$, b) $0.3 \mathrm{M}$, and c) $3 \mathrm{M}$ etidronic acid solutions at 273-333 K.

Figure 3 Changes in the time to reach the plateau voltage, $t_{\text {pla }}$, with the current density, $i_{\mathrm{a}}$, during galvanostatic anodizing in a) $0.03 \mathrm{M}$, b) $0.3 \mathrm{M}$, and c) $3 \mathrm{M}$ etidronic acid solutions at $273-333 \mathrm{~K}$.

Figure 4 a) SEM images of the aluminum dimple array formed by galvanostatic anodizing in a $0.3 \mathrm{M}$ etidronic acid solution at $293 \mathrm{~K}$ and $10-20 \mathrm{Am}^{-2}$. b) Normalized cell size histograms of the porous alumina calculated from the SEM images.

Figure 5 Changes in the average cell size, $D_{\text {c-ave, }}$ with the anodizing voltage, $U_{\text {a }}$, during galvanostatic anodizing in 0.03-3 $\mathrm{M}$ etidronic acid at 273-333 $\mathrm{K}$ and $0.25-350 \mathrm{Am}^{-2}$. The dashed line represents a linear relation calculated by the least-squares method.

Figure 6 Changes in the maximum cell size, $D_{\text {c-max }}$, and the minimum cell size, $D_{\text {c-min, }}$ with the anodizing voltage, $U_{\mathrm{a}}$, during galvanostatic anodizing in 0.03-3 $\mathrm{M}$ etidronic acid at $273-333 \mathrm{~K}$ and $0.25-350 \mathrm{Am}^{-2}$. The dashed lines represent a linear relation calculated by the least-squares method.

Figure 7 Changes in the number density of the cell formed in the porous alumina, $N_{\mathrm{c}}$, with the anodizing voltage, $U_{\mathrm{a}}$, during galvanostatic anodizing in 0.03-3 $\mathrm{M}$ etidronic acid at $273-333 \mathrm{~K}$ and $0.25-350 \mathrm{Am}^{-2}$. The dashed line represents the theoretical curve of ordered porous alumina with an ideal honeycomb distribution.

Figure 8 Changes in the maximum voltage, $U_{\max }$, with the anodizing voltage, $U_{\mathrm{a}}$, during galvanostatic anodizing in 0.03-3 $\mathrm{M}$ etidronic acid at 273-333 $\mathrm{K}$ and $0.25-350 \mathrm{Am}^{-2}$. The dashed line represents a linear relation calculated by the least-squares method. 


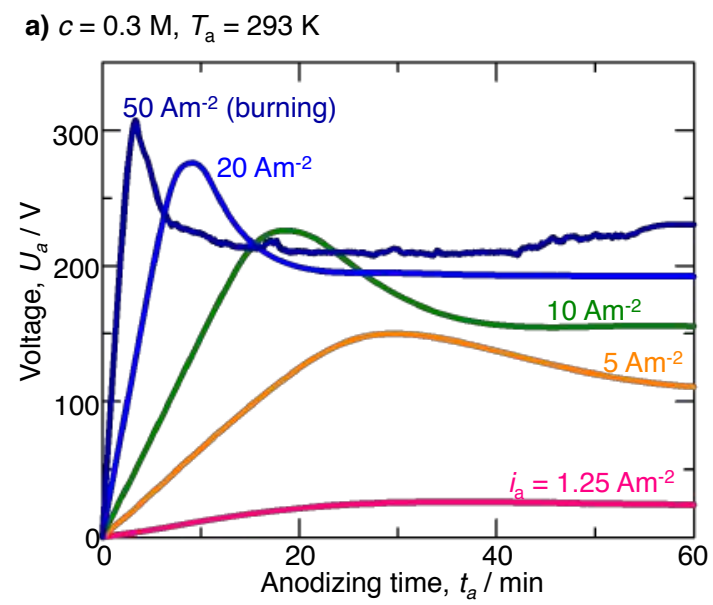

b) $i_{\mathrm{a}}=10 \mathrm{Am}^{-2}$

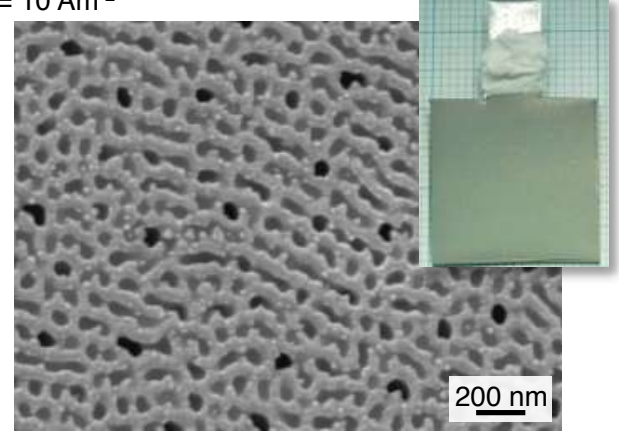

c) $i_{\mathrm{a}}=50 \mathrm{Am}^{-2}$

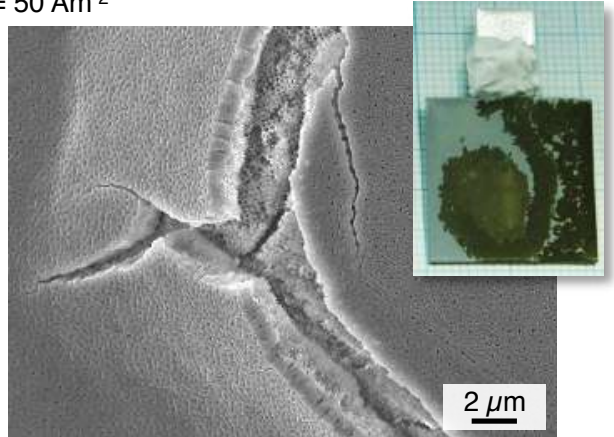

Figure 1. 


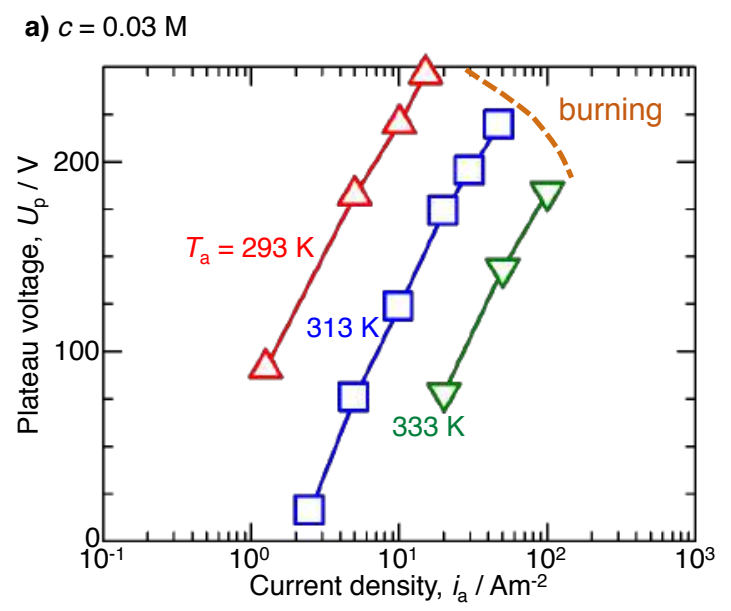

b) $c=0.3 \mathrm{M}$

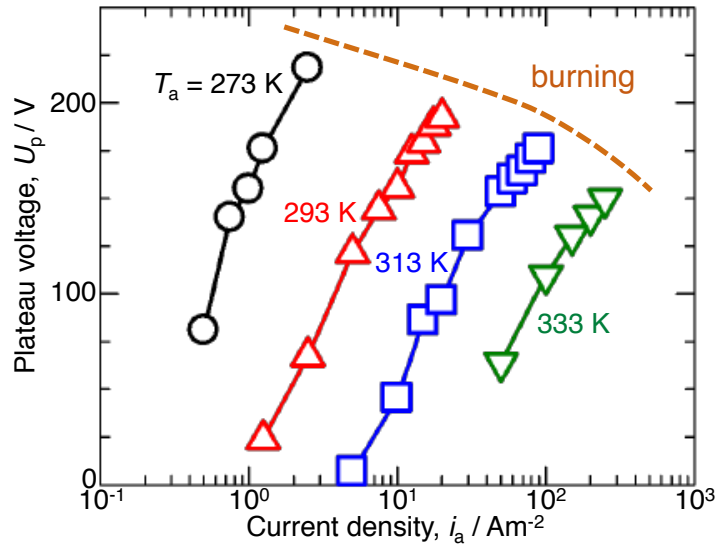

c) $c=3 \mathrm{M}$

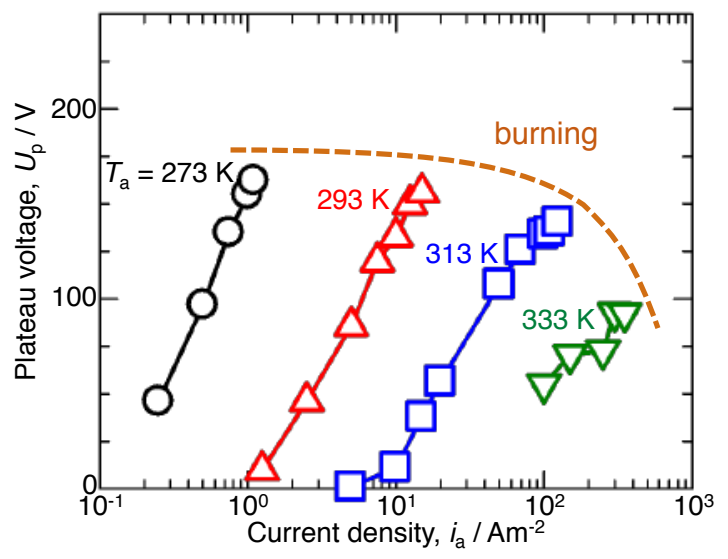

Figure 2. 

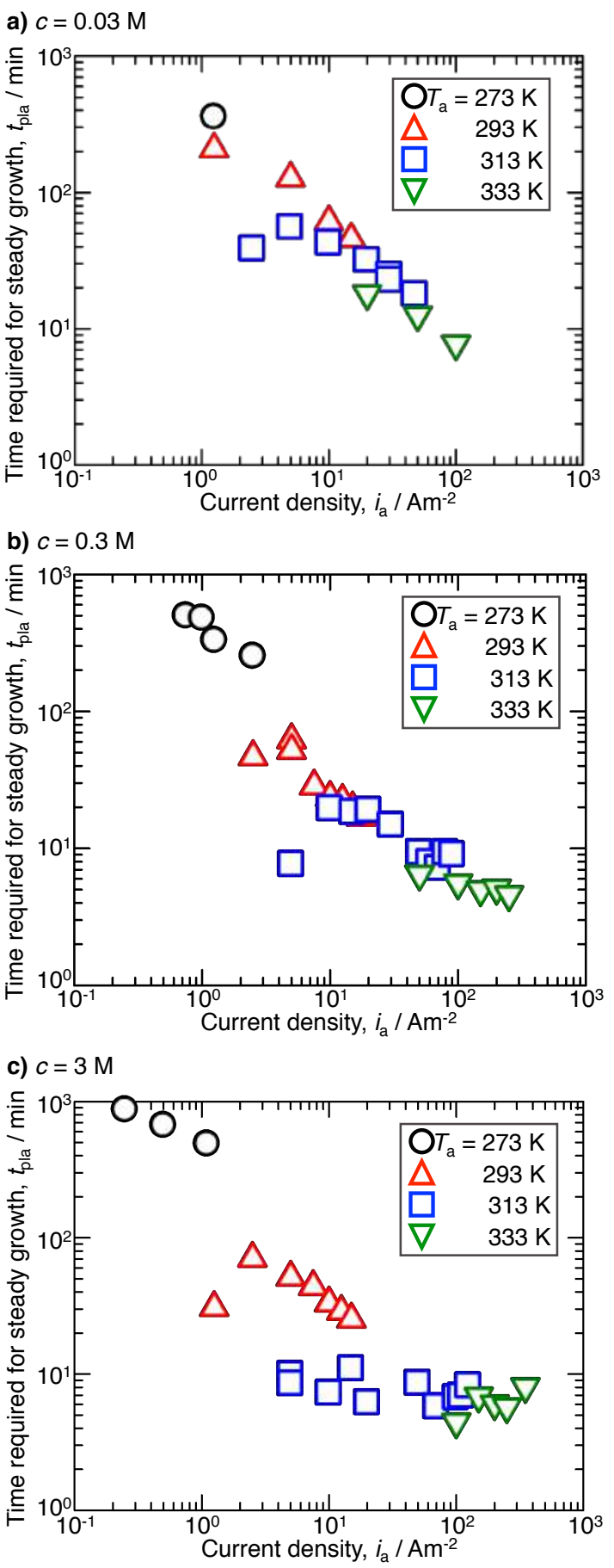

Figure 3. 
a) $i_{\mathrm{a}}=10 \mathrm{Am}^{-2}$

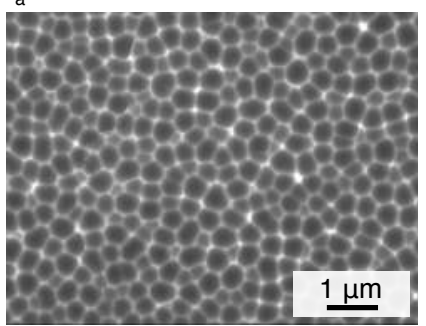

b)

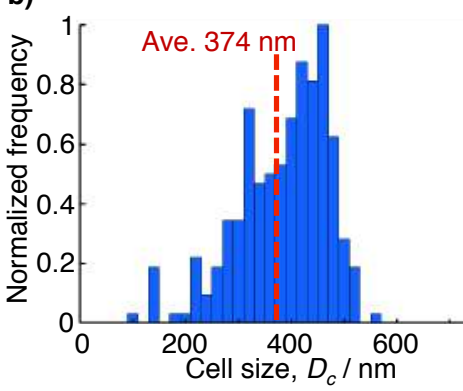

$i_{\mathrm{a}}=15 \mathrm{Am}^{-2}$

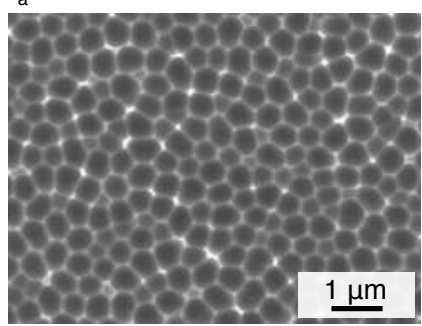

$1 \mu \mathrm{m}$ $i_{\mathrm{a}}=20 \mathrm{Am}^{-2}$
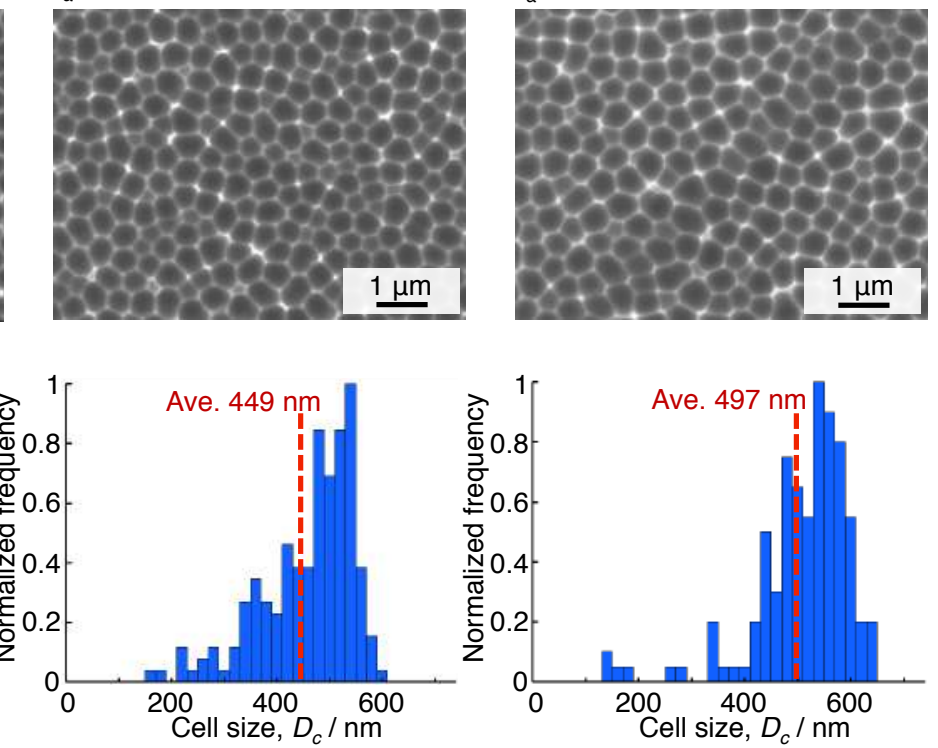

Figure 4. 
a) $c=0.03 \mathrm{M}$

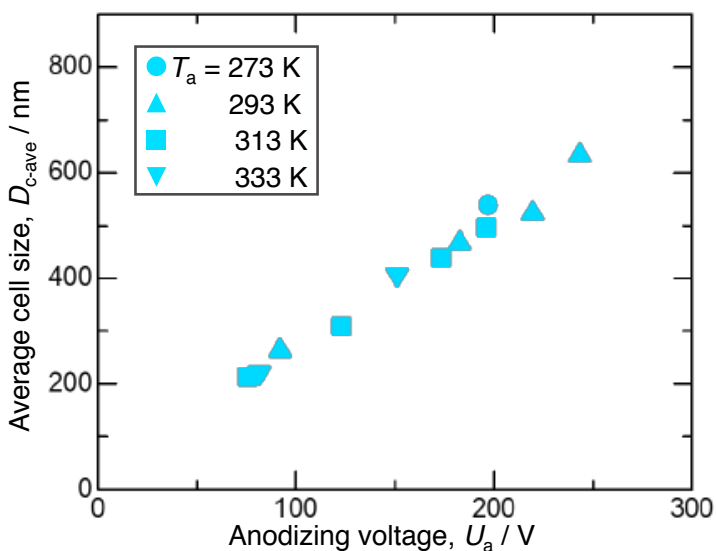

c) $c=3 \mathrm{M}$

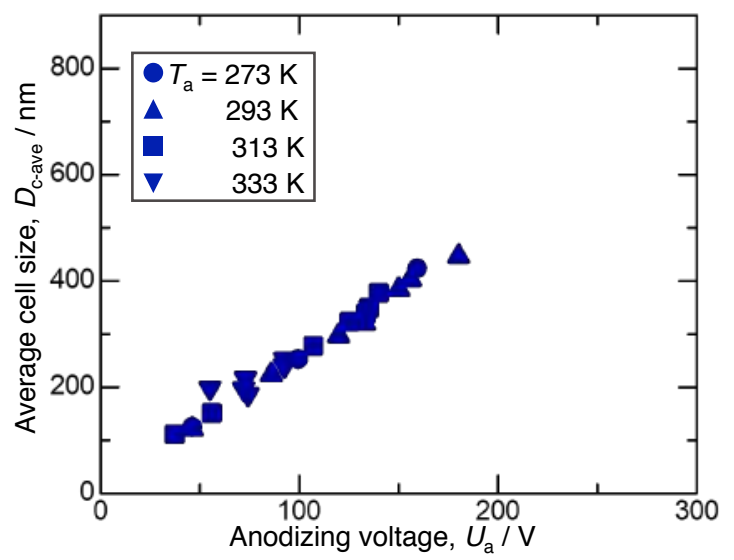

b) $c=0.3 \mathrm{M}$

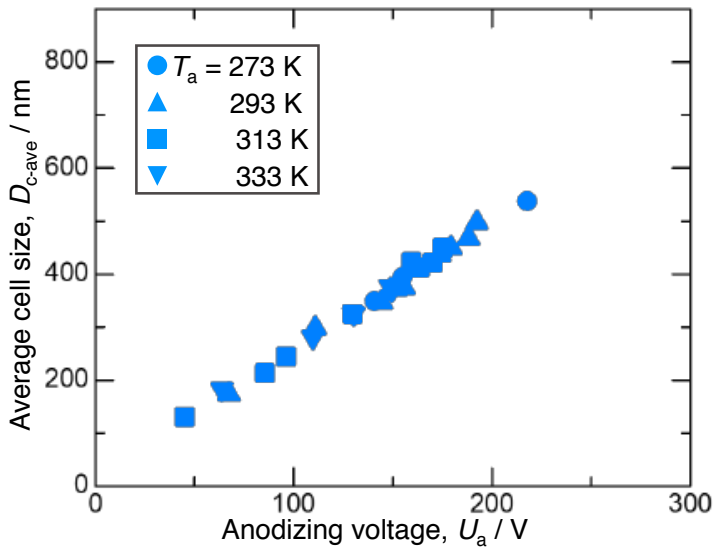

d) $c=0.03-3 \mathrm{M}$

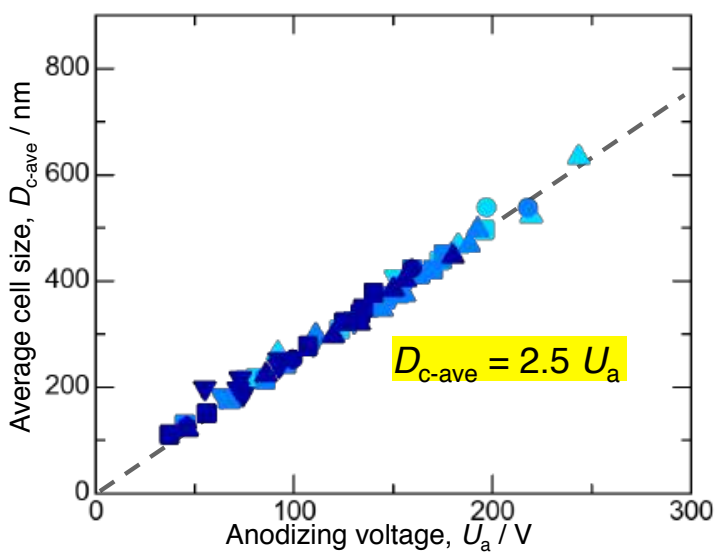

Figure 5. 
a) $c=0.03 \mathrm{M}$

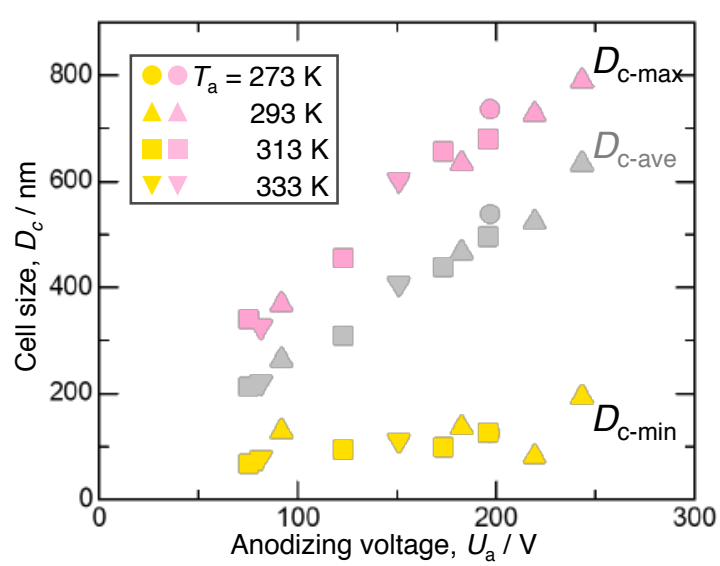

c) $c=3 \mathrm{M}$

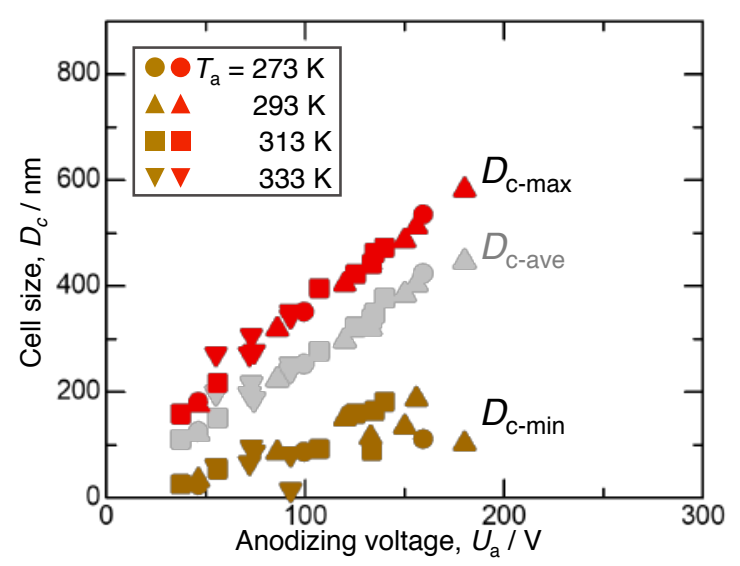

b) $c=0.3 \mathrm{M}$

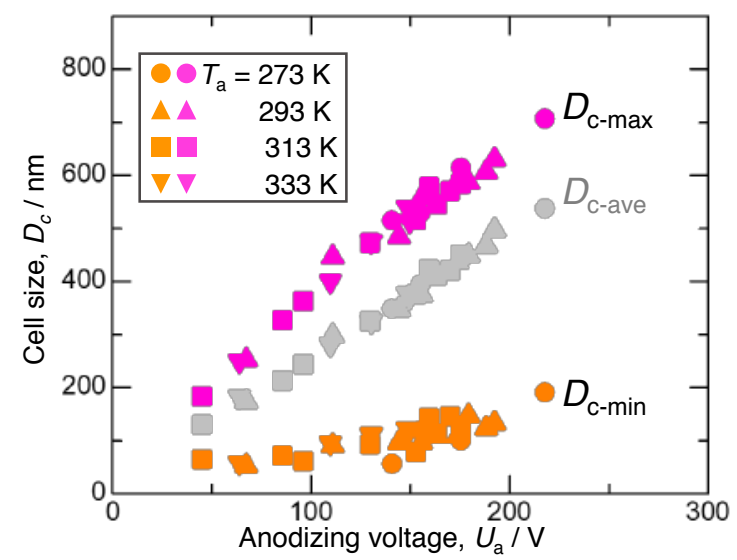

d) $c=0.03-3 \mathrm{M}$

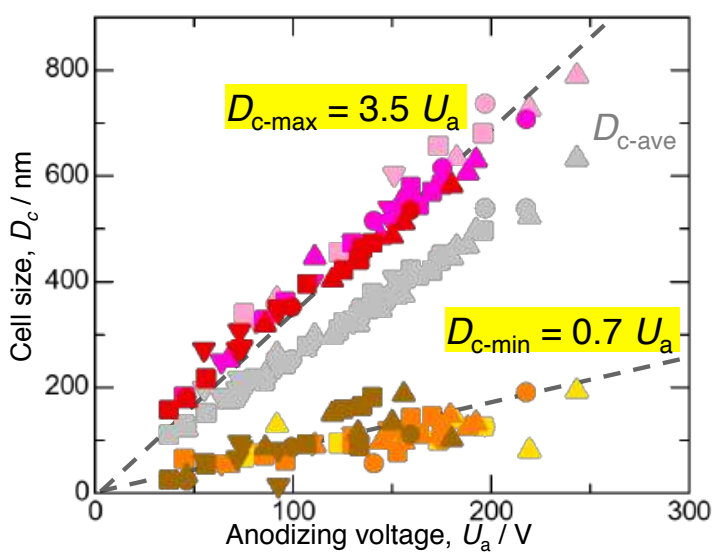

Figure 6. 

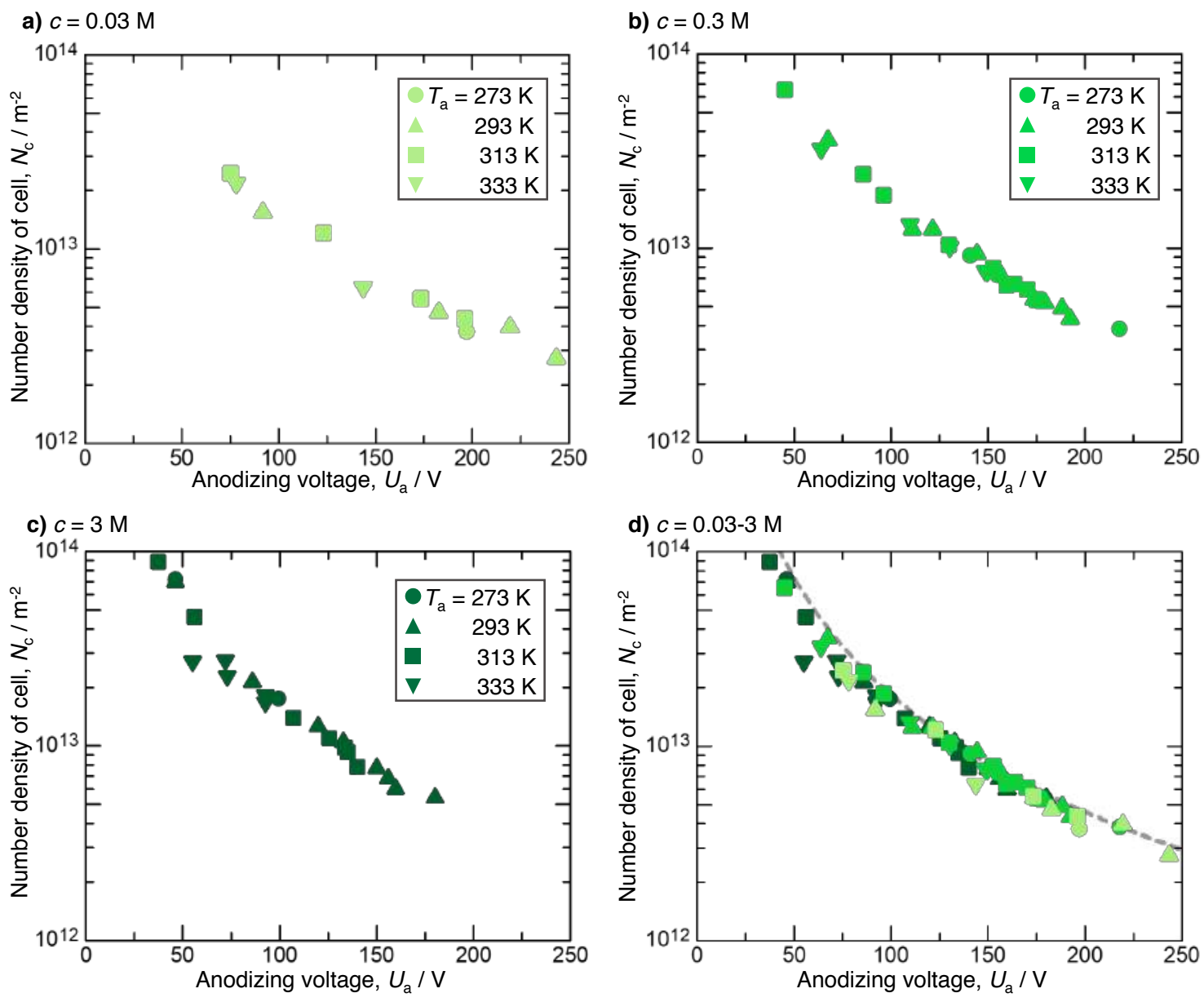

Figure 7. 
a) $c=0.03 \mathrm{M}$

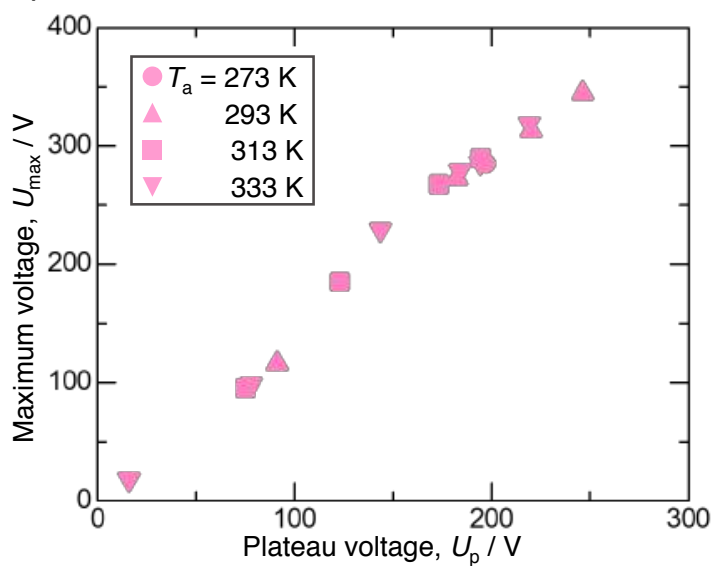

c) $c=3 \mathrm{M}$

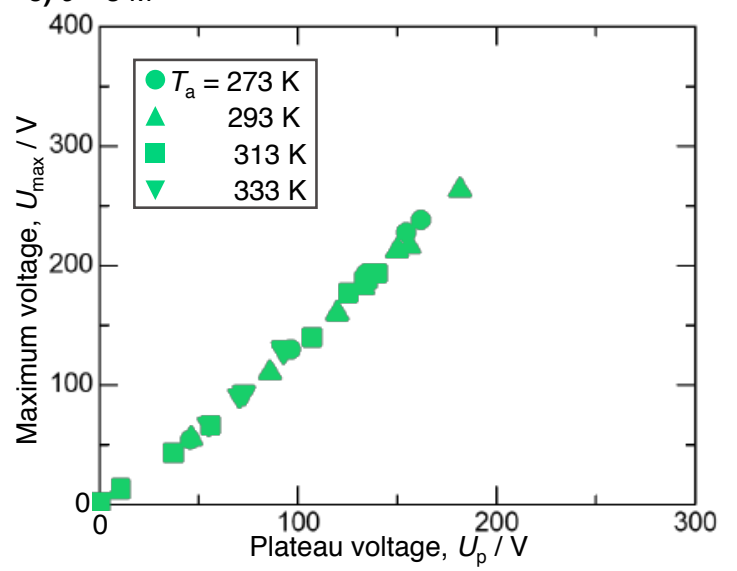

b) $c=0.3 \mathrm{M}$

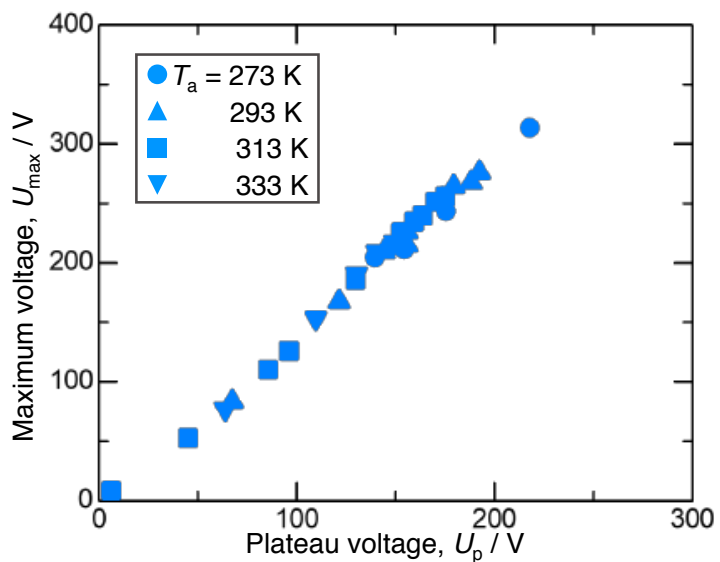

d) $c=0.03-3 \mathrm{M}$

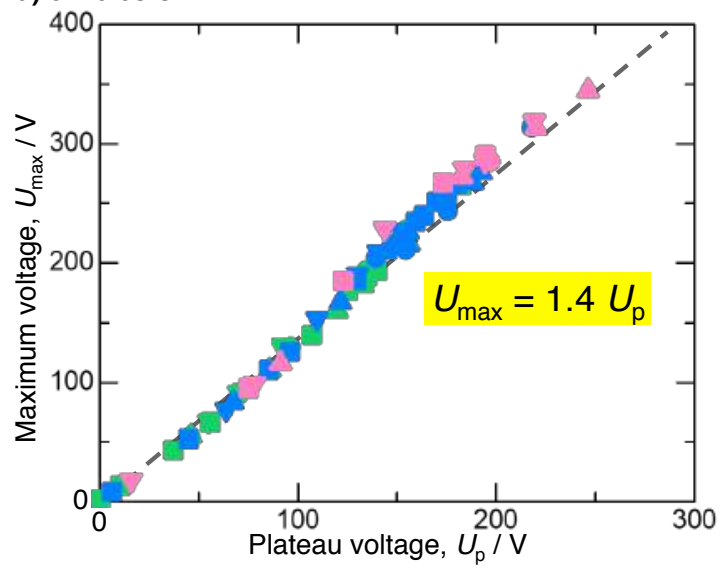

Figure 8. 